\title{
Control reset para maniobra de cambio de carril y validación con CarSim
}

\author{
Miguel Cerdeira, Pablo Falcón, Antonio Barreiro, Emma Delgado, Miguel Díaz-Cacho \\ Dpto. Enxeñería de Sistemas e Automática \\ Escola de Enxeñería Industrial \\ Universidade de Vigo, Galicia, Spain
}

\section{Resumen}

Se estudia la aplicación de control reset a una maniobra de cambio de carril comparándola con una técnica de control lineal convencional. Los resultados obtenidos atestiguan la capacidad de este tipo de control no lineal para superar en rendimiento a los controladores lineales afectados por las limitaciones fundamentales. Se ha comprobado la estabilidad del sistema tanto analíticamente como por simulación con el software de modelado dinámico CarSim.

Palabras clave: Maniobra de cambio de carril, Control reset, Condición $H_{\beta}$, CarSim.

\section{INTRODUCCIÓN}

Con el advenimiento progresivo de los vehículos autónomos ha surgido la necesidad de automatizar toda una batería de maniobras con el fin de dotar a los vehículos de absoluta funcionalidad para la variedad de situaciones que deben enfrentar. En este artículo se aborda el uso de control reset en una de estas maniobras, la de cambio de carril. Ésta, es una de las estudiadas más concienzudamente como atestiguan los numerosos artículos existentes, por ejemplo, [1], [2], [3], [4] que presentan distintas técnicas de control con el mismo objetivo, realizar el cambio de carril de manera segura y eficiente.

Los controladores reseteados son sencillamente reguladores estándar dotados con un mecanismo que reinicia a cero o a un determinado porcentaje uno o varios estados del controlador, siempre que se cumpla una determinada condición. La primera aplicación que trata el control reset está recogida en el trabajo de J. Clegg [5]. En este trabajo, Clegg demostró las ventajas de este tipo de control comparado con uno lineal. No obstante, a pesar de su utilidad, su estudio fue abandonado hasta los años 70, momento en el que el grupo de investigación de Horowitz lo recuperó [6, 7]. En estos artículos, se resaltó cómo el control reset ayuda a superar las limitaciones fundamentales que afectan a los sistemas lineales [8, 9]. Después de la publicación de estos artículos, el estudio del con- trol reset fue abandonado hasta los años 90, momento a partir del cuál el número de grupos de investigación interesados en esta materia aumentó considerablemente. Una de las propuestas más interesantes presentadas recientemente es el PI+CI [10] que combina las ventajas del controlador PI con las del integrador de Clegg. En los últimos años, el control reset se ha empleado en diferentes aplicaciones tal y como se muestra en la siguiente monografía [11]. Aparte de las aplicaciones recogidas en [11], existen varios artículos que utilizan esta técnica de control no lineal en diversas aplicaciones vehiculares. En [12] se emplea un controlador reset para la reconfiguración de pelotones de vehículos estudiando como éste ayuda a superar las limitaciones fundamentales. [13] aborda el uso de esta técnica de control para una aplicación de control de crucero adaptativo. Otros ejemplos $[14,15]$ incluyen el estudio del efecto de control reset sobre un sistema de frenado ABS.

El objetivo de este trabajo es explorar el potencial del control reset en una maniobra de cambio de carril. Para tal propósito, se considera un escenario idealizado y un modelo cinemático sencillo para centrar el estudio en cómo el control reset mejora la maniobra. Para probar la estabilidad de todo el sistema (controlador reset \& planta linealizada) se emplea una condición conocida como $H_{\beta}$ basada en una función de Lyapunov cuadrática que debe ser decreciente en todo el espacio de estados y no incrementar en los instantes de salto [16].

Para la validación del modelo empleado, así como del controlador desarrollado, se recurre al software comercial CarSim desarrollado por Mechanical Simulation Corporation.

Este artículo está organizado de la siguiente manera. En la Sección 2, se presenta el modelo empleado para la maniobra de cambio de carril así como la validación del mismo con CarSim. A continuación, en el apartado 3 se exponen todas las consideraciones utilizadas para el diseño del controlador y se presentan resultados sobre la estabilidad del sistema. Por último, en la sección 4, se muestran los resultados de las simulaciones para finalmente presentar las conclusiones en la sección 5 . 
Tabla 1: Parámetros del modelo de vehículo

lf Distancia desde el eje delantero al CM

lr Distancia desde el eje trasero al CM

y Posición lateral del vehículo

$v_{0} \quad$ Velocidad longitudinal del vehículo

$\psi \quad$ Guiñada

$\delta \quad$ Ángulo de las ruedas

\section{MODELADO Y VALIDACIÓN}

\subsection{MODELO BICICLETA CINEMÁTICO}

Para el modelado de la maniobra del vehículo se ha empleado el modelo bicicleta cinemático, representado en la Fig. 1 y cuyos parámetros están recogidos en la Tabla 1. La descripción detallada del modelo puede ser encontrada en [17]. Se denomina modelo bicicleta ya que se trabaja con la simplificación de que tanto las ruedas delanteras como las traseras están concentradas en el punto medio de cada uno de los dos ejes. Este modelo es adecuado para velocidades pequeñas [18]. Ya que se pretende que el controlador desarrollado trabaje a mayores velocidades, y por los motivos que se dan en el apartado de validación del modelo, habrá que modificar ligeramente el modelo para obtener resultados válidos. Otra simplificación que se ha tenido en cuenta es que al trabajar con ángulos de volante pequeños, puede linealizarse el modelo en torno al punto operativo del controlador. Las ecuaciones del modelo cinemático bicicleta en el espacio de estados pueden verse en (1) en donde $y$ y $\psi$ son los estados, $\delta$ es la actuación, $Y$ es la salida que en este caso es igual al estado y y los coeficientes $b 1=\frac{l f * v_{0}}{l f+l r}$ y $b_{2}=\frac{v_{0}}{l f+l r}$.

$$
\left\{\begin{array}{l}
\left(\begin{array}{c}
\dot{y} \\
\dot{\psi}
\end{array}\right)=\left(\begin{array}{cc}
0 & v_{0} \\
0 & 0
\end{array}\right)\left(\begin{array}{l}
y \\
\psi
\end{array}\right)+\left(\begin{array}{l}
b_{1} \\
b_{2}
\end{array}\right) \delta \\
Y=\left(\begin{array}{ll}
1 & 0
\end{array}\right)\left(\begin{array}{c}
y \\
\psi
\end{array}\right)+0 * \delta
\end{array}\right.
$$

A partir de estas ecuaciones se puede obtener que la función de transferencia entre la entrada de actuación (ángulo de las ruedas delanteras) y la salida (posición lateral del centro de masas del vehículo CM) es igual a:

$G_{\text {teorica }}(s)=\frac{y(s)}{\delta(s)}=\frac{b_{1} s+v_{0} b_{2}}{s^{2}}=\frac{\frac{l f * v_{0}}{l f+l r} s+\frac{v_{0}^{2}}{l f+l r}}{s^{2}}$

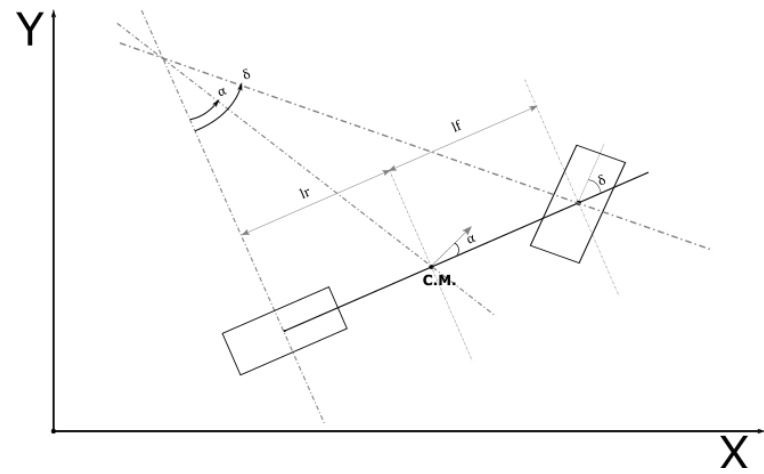

Figura 1: Modelo bicicleta cinemático.

\subsection{VALIDACIÓN DEL MODELO CON CARSIM}

Para la validación del modelo empleado en este trabajo se utilizó el software de simulación CarSim [19]. Este software es utilizado por numerosos fabricantes (OEMs) y suministradores de componentes del automóvil (Tier 1), universidades y centros de investigación de todo el mundo, siendo un estándar de la industria del automóvil. Existen varios trabajos en donde se puede observar que este software es útil en tareas de validación de modelos [20] y simulación de tráfico rodado [21].

La validación del modelo se ha realizado mediante la comparación del comportamiento del modelo propuesto en (1) frente a la respuesta obtenida del modelo interno de CarSim. El modelo interno que emplea CarSim es un modelo muy complejo que incluye por ejemplo la dinámica del vehículo, numerosas variables internas de los distintos componentes, tiempos de comunicaciones y actuación o el entorno del vehículo. En este caso, se trata de simplificar este complejo modelo en un modelo más sencillo de utilizar en el que se pueda sintonizar un controlador efectivo para la maniobra de cambio de carril. Primeramente es necesario particularizar el modelo propuesto para el coche escogido, en este caso se seleccionó un coche tipo Sedan clase D (ver Fig. 2).

Partiendo de (2), el modelo del Sedan viene dado por las características físicas del coche (lf y $l r$, ver Tabla 1) y la velocidad de crucero del vehículo $v_{0}$. En este caso los parámetros son: $l f=1,110 \mathrm{~m}$, $l r=1,670 m$ y $v_{0}=90 \mathrm{~km} / \mathrm{h}(25 \mathrm{~m} / \mathrm{s})$. Con estos valores, el modelo teórico del vehículo resultante es:

$$
G_{\text {teorica }}(s)=\frac{9,982 s+224,8}{s^{2}}
$$

Después de analizar varias pruebas, se ha compro- 


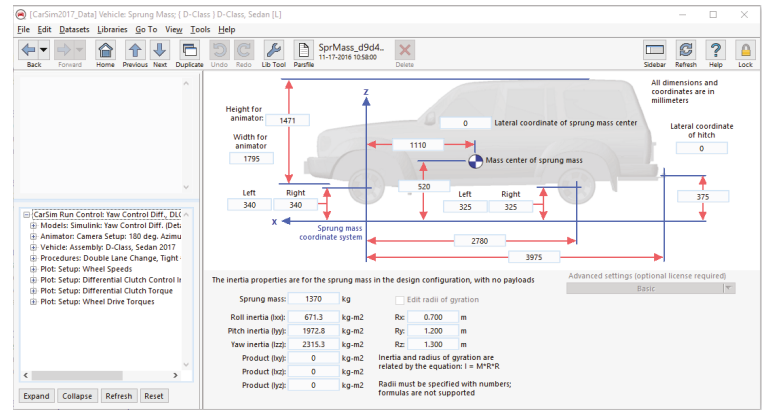

Figura 2: Vista de la pantalla de configuración del vehículo en CarSim.

bado que el modelo de CarSim se puede ajustar al modelo descrito en (1) con la salvedad de que existe un cierto adelanto entre los datos obtenidos con el modelo propuesto y los datos obtenidos directamente con el modelo interno de CarSim. Esto es debido en gran medida a que el modelo cinemático es válido a baja velocidad, mientras que a $v_{0}=90$ $\mathrm{km} / \mathrm{h}$ aparecen otros efectos dinámicos. Por ello, se debe añadir un atraso en el modelo propuesto para ajustar mejor los datos obtenidos, este atraso puede ser aproximado por un primer orden con una constante de tiempo $\tau$. Entonces el modelo propuesto completo es el descrito en (4).

$$
G(s)=\frac{b_{1} s+b_{2} v_{0}}{s^{2}} \times \frac{1}{\tau s+1}
$$

El experimento realizado para la identificación de la planta se muestra en la Fig. 3. Como se puede observar en dicha figura, el experimento se trata de una maniobra controlada de cambio de carril en donde se modifica la consigna de posición lateral del vehículo mediante una entrada tipo escalón. La adquisición de los datos de la planta se realizó entre las señales $u$ e $y$ correspondientes a la entrada y la salida de la planta. Este experimento está basado en señales entrada-salida que se generan en una maniobra típica de cambio de carril. El bloque correspondiente al modelo de CarSim recibe entrada de giro de ruedas en grados y la salida es la posición lateral del centro de masas del vehículo.

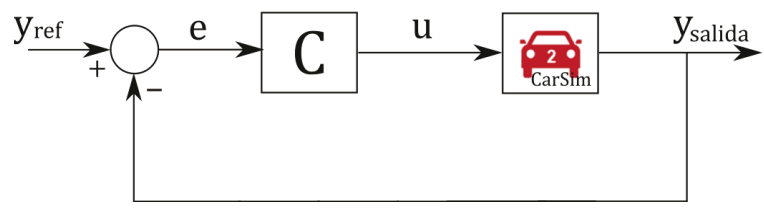

Figura 3: Sistema dispuesto para la identificación.

Se ha identificado la planta mediante el barrido de los parámetros de la función de transferencia sobre los datos adquiridos en el simulador CarSim. Por las características físicas del vehículo la solución debe tener unos valores semejantes a la función de transferencia teórica. El resultado de la identificación se muestra en (5). El error cuadrático medio de la planta modelada y los datos adquiridos de la planta real es de $7,810 \times 10^{-3}$ metros, por lo que se puede considerar representativo de la planta real.

$$
G(s)=\frac{8,3 s+169,8}{0,19 s^{3}+s^{2}}
$$

O lo que viene a ser, empleando la forma descompuesta en planta más retraso de actuación:

$$
G(s)=\frac{8,3 s+169,8}{s^{2}} \times \frac{1}{0,19 s+1}
$$

En la Fig. 4 se observa la correspondencia que existe entre los datos extraídos de la respuesta temporal del modelo identificado y los datos obtenidos del modelo interno de CarSim. Se puede decir que para una velocidad $v_{0}=90 \mathrm{~km} / \mathrm{h}$ el modelo identificado es válido para el vehículo escogido y se puede proceder al diseño de un controlador apto para los experimentos con CarSim.

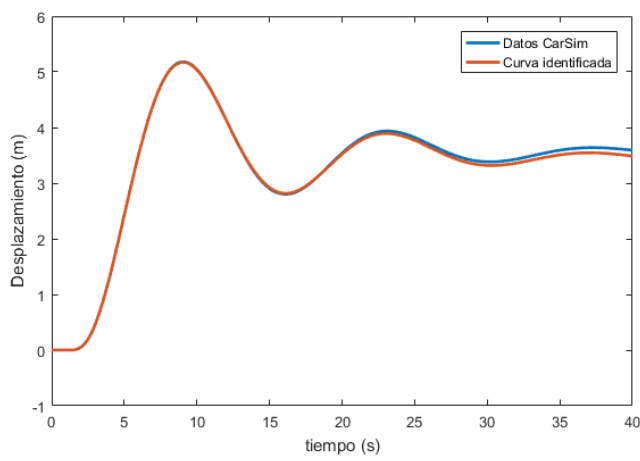

Figura 4: Comparación entre el modelo identificado y el modelo interno de CarSim.

\section{CONTROL Y ESTABILIDAD}

\subsection{CONTROL}

Tal como se mencionó en el apartado introductorio, el propósito de este artículo es explorar las ventajas potenciales del control reset en la maniobra de cambio de carril. Un controlador reset, con entrada $e(t)$ y salida $u(t)$, viene dado por $(7)$ donde $A_{r}$ es una matriz diagonal con unos en los estados que no son reseteados. 


$$
\begin{cases}\dot{x}_{r}(t)=A_{r} x(t)+B_{r} e(t) & \text { si } e(t) \neq 0 \\ x_{r}\left(t^{+}\right)=A_{\rho} x(t) & \text { si } e(t)=0 \\ u(t)=C_{r} x_{r}(t)+D_{r} e(t) & \end{cases}
$$

Este tipo de controlador se comporta exactamente igual que un controlador lineal hasta que se da la condición de reinicio, siendo ésta normalmente el cruce por cero de la señal de error. Al controlador lineal al que se le dota del mecanismo de reinicio se le conoce como controlador lineal base. Para explotar el potencial del reinicio al máximo se deben considerar una serie de especificaciones de diseño para el controlador lineal base. Como es obvio, el controlador base debe presentar sobreoscilación para que se dispare el mecanismo de reinicio y su efecto surta efecto. [10] recoge una colección de reglas de sintonía para facilitar la obtención de un controlador reseteado óptimo.

Al contrario que los controladores lineales, los controladores reseteados no están afectados por las llamadas limitaciones lineales fundamentales. Por ejemplo, cuando en bucle abierto, nuestro sistema presenta un integrador, ha quedado probado que independientemente del regulador escogido, la señal de error está restringida por la igualdad $\int_{0}^{\infty} e(\tau) d \tau=1 / K_{v}[22]$, donde $K_{v}$ es la ganancia de velocidad. La planta (6) para control lateral contiene un doble integrador y otras raíces, con lo que $K_{v}=\infty$. En este caso, cualquier controlador lineal estará afectado por la siguiente restricción:

$$
\int_{0}^{\infty} e(\tau)=0
$$

Los controladores reseteados, al no verse afectados por esta limitación, y, siempre que hayan sido bien sintonizados, proporcionan mejores respuestas que cualquier controlador lineal para los objetivos de diseño fijados. La justificación completa de como un controlador reseteado de idénticas características al empleado en este artículo produce una respuesta con un mejor régimen transitorio puede ser encontrada en [12].

Para el diseño del controlador lineal base, se ha partido del controlador desarrollado en [12] cuya respuesta temporal es idónea para esta aplicación. Para ello hay que aplicar dos adaptaciones. La primera es convertir la planta en $1 / s^{2}$ como en [12]. Dado que el cero y el polo de (6) son bien conocidos, esto se puede conseguir con un bloque de prefiltrado $F(s)$ (9) que cancela dichas raíces, ver Fig. 5.

$$
F(s)=\frac{0,19 s+1}{8,3 s+169,8}
$$

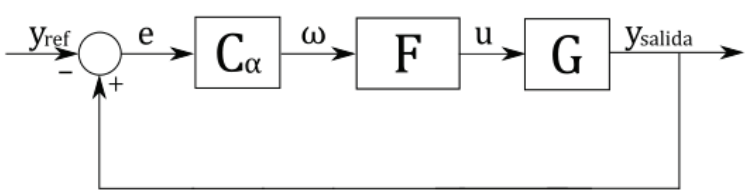

Figura 5: Lazo de control.

Obteniéndose de este modo una planta $P(s)=$ $F(s) * G(s)$ equivalente a $1 / s^{2}$.

La segunda adaptación es incorporar en el controlador de [12] un par de parámetros adicionales $k$ y $\alpha$, que confieren flexibilidad al diseño.

La función de transferencia de controlador de partida de [12] es (10).

$$
C(s)=\frac{2(s+0,01)}{(s+0,5)(s+2)(s+3)}
$$

Y el controlador propuesto es (11).

$$
C_{\alpha}(s)=\frac{k *\left(\frac{s}{\alpha}+0,01\right)}{\left(\frac{s}{\alpha}+0,5\right)\left(\frac{s}{\alpha}+2\right)\left(\frac{s}{\alpha}+3\right)}
$$

Primeramente se debe relacionar la sobreoscilación del controlador con la ganancia que se introduce a través del parámetro $k$, esto es importante para restringir la amplitud máxima de posición lateral del vehículo y que éste no salga de los límites del carril.

En segundo lugar, a través del parámetro $\alpha$ se puede modificar la respuesta temporal del controlador haciendo que los requerimientos de aceleración y jerk máximos no superen las limitaciones de confort. En [23] se establece un límite para aceleración lateral de $0,05 \mathrm{~g}\left[\mathrm{~m} / \mathrm{s}^{2}\right]$ y un límite de jerk lateral de $0,1 g\left[\mathrm{~m} / \mathrm{s}^{3}\right]$, siendo $g$ el módulo de la aceleración de la gravedad.

Si se parte de una señal en el dominio de Laplace tal que $D(s)$ que equivale a $d(t)$ en dominio temporal y se aplica el factor $\alpha>1$ de la forma que la señal sea $D(s / \alpha)$, entonces la respuesta temporal es $d(\alpha t)$, lo que hace que la señal resultante sea $\alpha$ veces más rápida. Al variar el valor de $\alpha$ se acelera $(\alpha>1)$ o ralentiza $(\alpha<1)$ la respuesta temporal del sistema, como se observa en la Fig. 6. Este cambio, al derivar usando la ley de la cadena, modifica la velocidad del sistema por $\alpha$, la aceleración por $\alpha^{2}$ y jerk por $\alpha^{3}$. Lo que permite ajustar el controlador y planta a los límites demandados de aceleración y jerk máximos.

Cabe destacar que la planta $P(s)$ se debe modificar del mismo modo que el controlador $C_{\alpha}$ siendo la nueva planta del sistema $P_{\alpha}(s)=1 /\left(s^{2} / \alpha^{2}\right)$. El coeficiente $\alpha^{2}$ será absorvido por el controlador, 


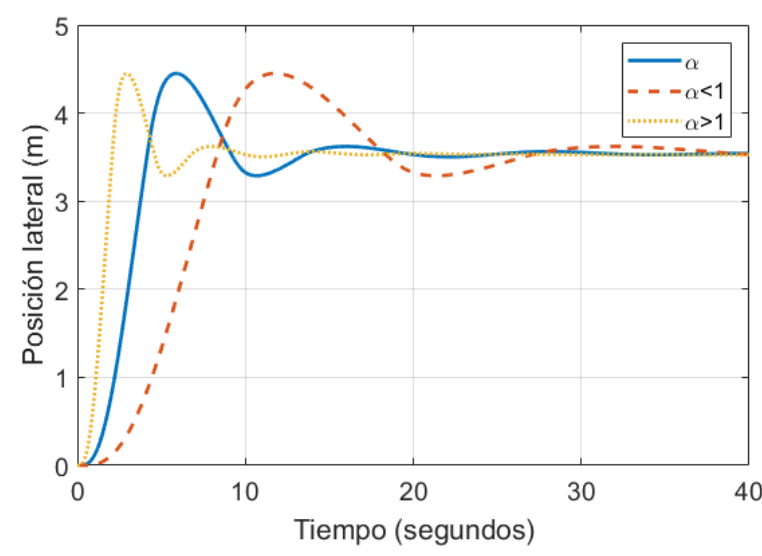

Figura 6: Respuestas obtenidas variando el valor de $\alpha$

como $P_{\alpha}=\alpha^{2} P$ entonces el controlador real será $C_{\alpha} \alpha^{2}$.

En el controlador presentado en [12], solamente uno de los tres estados del controlador era reseteado. En nuestro controlador modificado, $s=$ $-0,5 * \alpha$ ha sido escogido por ser el más próximo al origen y por tanto, el más lento. Se separa por tanto el elemento $\frac{1}{s / \alpha+0,5}$ y se constituye lo que se conoce como First Order Reset Element (FORE) cuyas ecuaciones pueden verse en (12) siendo $\zeta$ la salida del FORE. De esta manera, el controlador puede ser expresado mediante $C_{\alpha}(s)=C_{1}(s) C_{2}(s)$ dónde $C_{1}(s)$ es la función de transferencia del FORE, parte no lineal, y $C_{2}(s)$ es la función de transferencia de la parte lineal.

$$
\left\{\begin{array}{lll}
\dot{\zeta}(t)=-0,5 \alpha \zeta(t)+\alpha e(t) & \text { si } & e \neq 0 \\
\zeta\left(t^{+}\right)=0 & \text { si } & e=0
\end{array}\right.
$$

En la Fig. 7 puede apreciarse cómo el FORE ha sido emplazado antes de la parte lineal para evitar que la salida del controlador sea directamente la salida del FORE y conseguir de esta manera que la salida final del controlador no presente saltos.

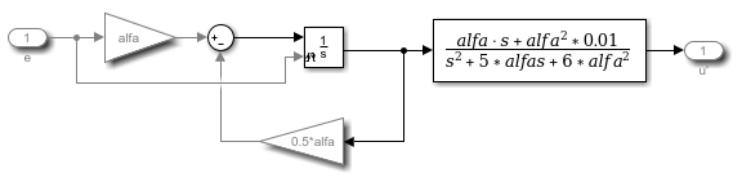

Figura 7: Controlador propuesto.

El controlador propuesto (11) adaptado con los parámetros $k=1,3$ y $\alpha=0,645$ proporciona la respuesta temporal mostrada en las Fig. 8, 9 y 10 de posición, aceleración y jerk respectivamente. Como se puede ver, los límites de aceleración y jerk laterales se cumplen para el sistema formado por el controlador ajustado $C_{\alpha}$ y la planta $P_{\alpha}$ pero no así para el sistema formado por el controlador de partida $C(s)$ y la planta $P(s)$.

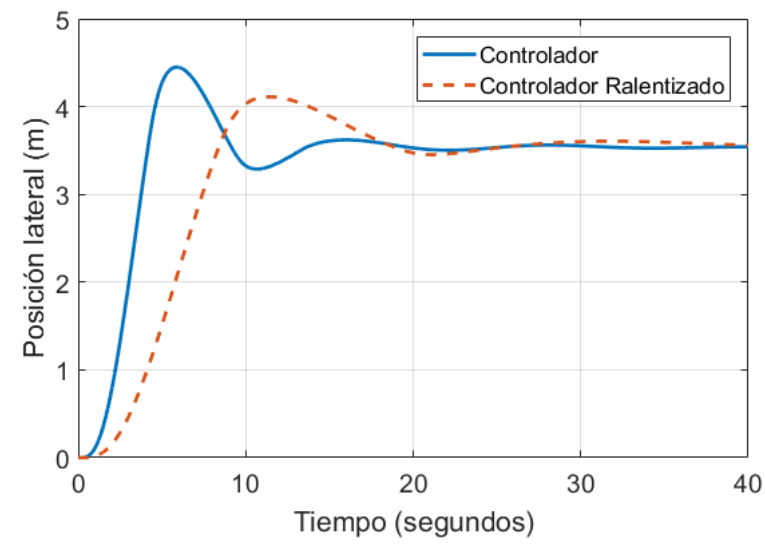

Figura 8: Comparativa de la posición lateral.

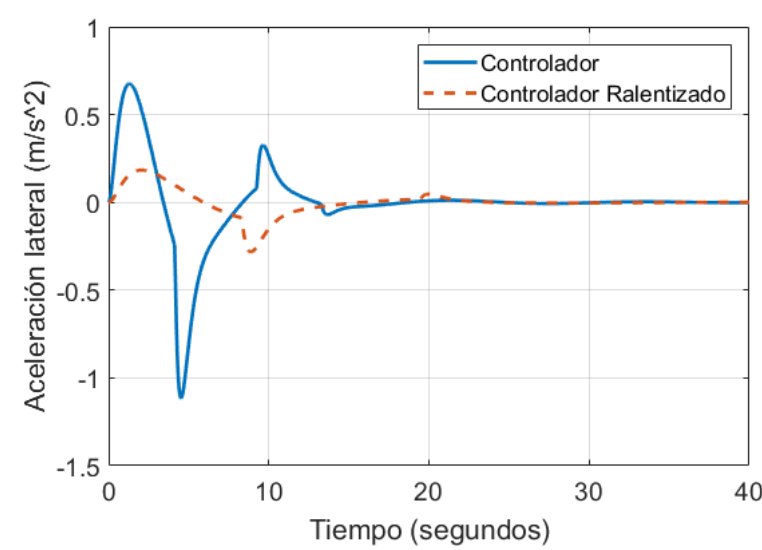

Figura 9: Comparativa de la aceleración lateral.

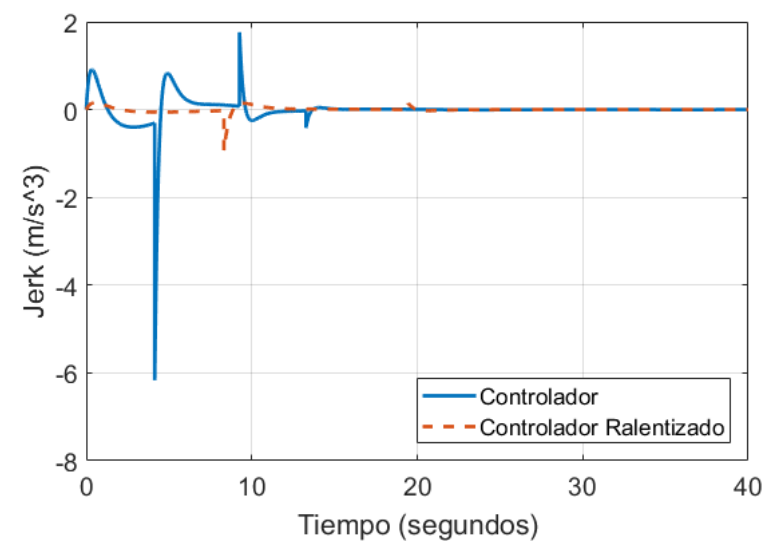

Figura 10: Comparativa de jerk lateral.

\subsection{ESTABILIDAD}

Para probar la estabilidad del sistema se ha empleado una condición conocida como $H_{\beta}$ definida 
para los sistemas con control reset. Así como fue presentado en la introducción, esta condición está basada en una función de Lyapunov cuadrática que debe ser decreciente en todo el espacio de estados conjuntamente con las trayectorias del sistema y no incrementar en los instantes de salto [16]. Se trata de una herramienta que permite comprobar la estabilidad fácilmente mediante el uso de LMIs o directamente a través de gráficas frecuenciales. Se ha optado en este artículo por el segundo método.

Para comprobar la condición $H_{\beta}$ se considera un sistema de control en lazo cerrado de estado $x=$ $\left(x_{p}^{\top}, x_{r}^{\top}\right)^{\top}$ de dimensión $n=n_{p}+n_{r}$, dado por:

$$
\left\{\begin{array}{lll}
\dot{x}=A x & \text { si } & e=C_{p} x_{p} \neq 0 \\
x_{r}\left(t^{+}\right)=0 & \text { si } & e=C_{p} x_{p}=0
\end{array}\right.
$$

Siendo la respuesta frecuencial $H_{\beta}(j \omega)\left(n_{r} \times n_{r}\right)$

$$
H_{\beta}(j \omega)=H(j \omega I-A)^{-1} B
$$

donde $H\left(n_{r} \times n\right)$ vale:

$$
H=\left(\beta C_{p}, P_{3}\right)
$$

y donde $B\left(n \times n_{r}\right)$ es:

$$
B=\left(0, I_{n_{r}}\right)^{\top}
$$

Entonces se prueba ([11], [16]) que el sistema de control es asintóticamente estable si $H_{\beta}(j \omega)$ es SPR (positiva real estricta).

En la Fig. 11 se ve como para $\beta=0,5$ se satisface $H_{\beta}$, es decir, para cualquier frecuencia se cumple que la parte real de los autovalores de la matriz $H(j \omega)$, que en este caso es escalar, siempre es positiva. Del cumplimiento de la condición se deduce que el sistema es cuadráticamente estable. Al mismo resultado se puede llegar por inspección directa del diagrama de Nyquist para $\beta=0,5$ (ver Fig. 12) ya que de ella se desprende que $H_{\beta}(j \omega)$ es estrictamente positiva.

\section{VALIDACIÓN DE LOS RESULTADOS CON CARSIM}

La validación del controlador de cambio de carril se realiza con el sistema planteado en la Fig. 5 mediante simulación sustituyendo el bloque $G(s)$ por el bloque de CarSim, ya mostrado en la Fig. 3.

Se ha planteado un experimento donde se dispone un vehículo tipo Sedan sobre una carretera en linea recta a una velocidad constante de $90 \mathrm{~km} / \mathrm{h}$,

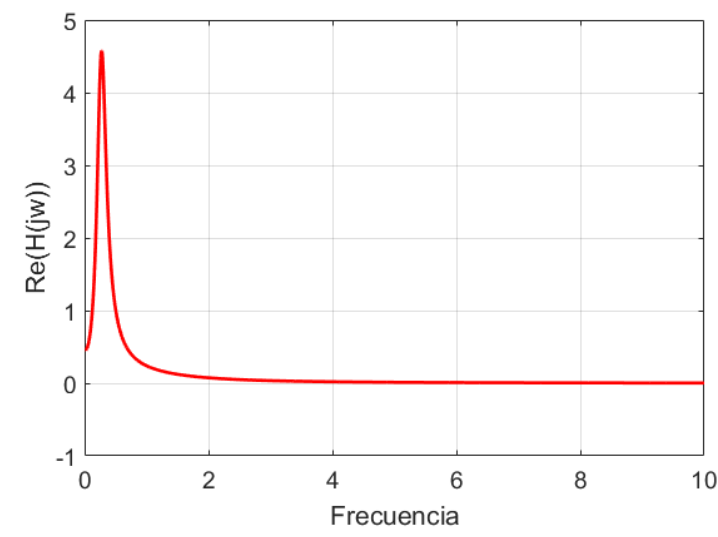

Figura 11: $\operatorname{Re}\left(H_{\beta}(j \omega)\right.$ vs $\omega$ para $\beta=0,5$

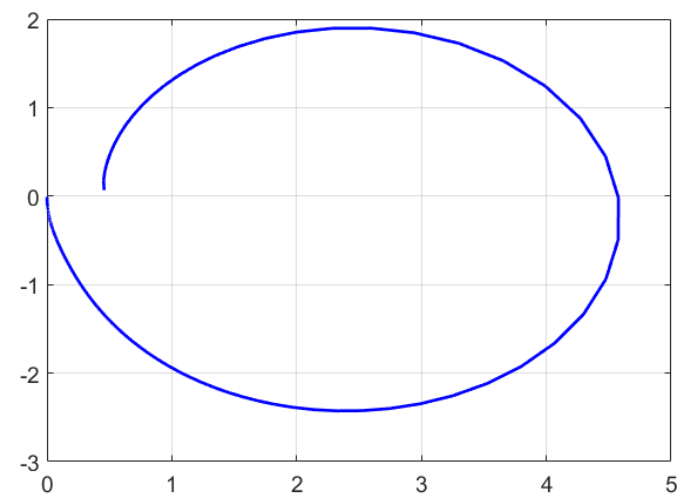

Figura 12: Nyquist de $H_{\beta}(j \omega)$ para $\beta=0,5$

y se aplica en $t=1 s$ un cambio de consigna tipo escalón de 3.5 metros que hace que el vehículo se desplace lateralmente y cambie de carril.

En el experimento se prueba la valía del control reset para la maniobra de cambio de carril en carretera directamente sobre CarSim. El controlador reseteado diseñado permite obtener mejores resultados frente a un controlador lineal convencional como se puede apreciar en la Fig. 13. Se muestra como el controlador reseteado es claramente mejor que el controlador lineal base. La clave de la propuesta es que también es mejor que cualquier otro controlador lineal, sometido a la limitación (8), para ciertos objetivos de diseño. Estos objetivos se detallan en [12], que combinan una fase de subida suave, sobreoscilación baja y establecimiento rápido, y solo son alcanzables con la estrategia de reset. Esta estrategia de control logra realizar el cambio de carril rápidamente evitando la sobreoscilación que se produce en el caso del controlador lineal. Además, las señales de actuación provenientes de los controladores no tienen valores excesivamente exigentes por lo que es perfectamente aplicable sobre el vehículo.

En las Fig. 15 y 16 se pueden comprobar los límites de confort de la aceleración lateral y el jerk 


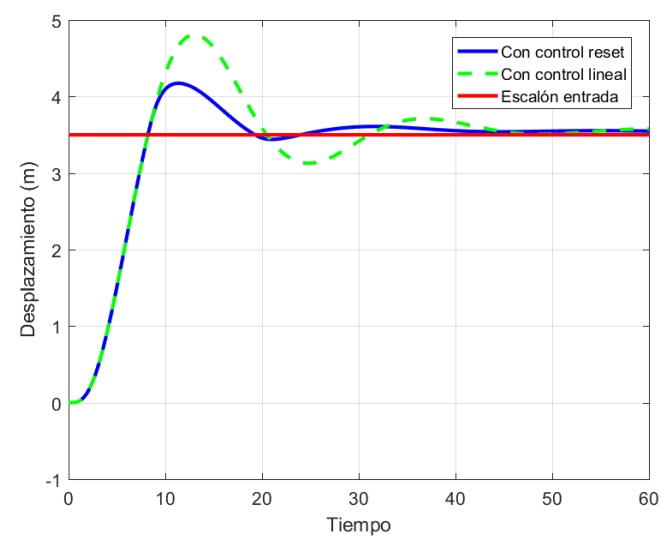

Figura 13: Respuesta del vehículo ante entrada escalón de 3.5 metros de desplazamiento lateral.

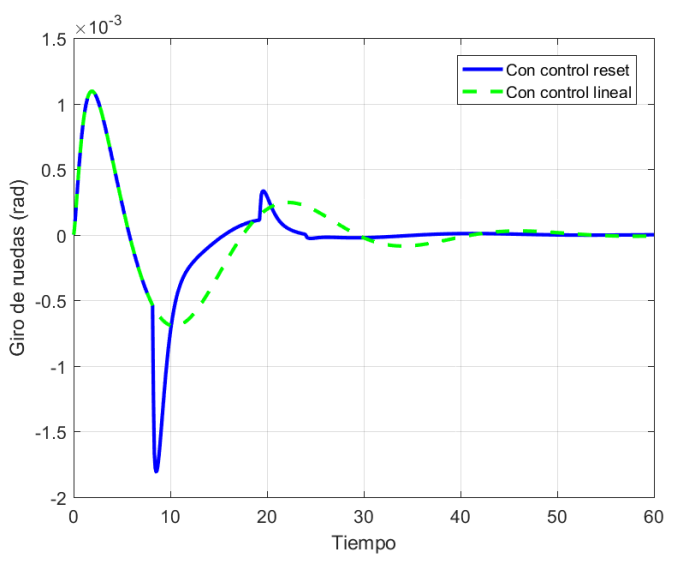

Figura 14: Señales de control aplicadas sobre el bloque CarSim, ángulo de ruedas en radianes.

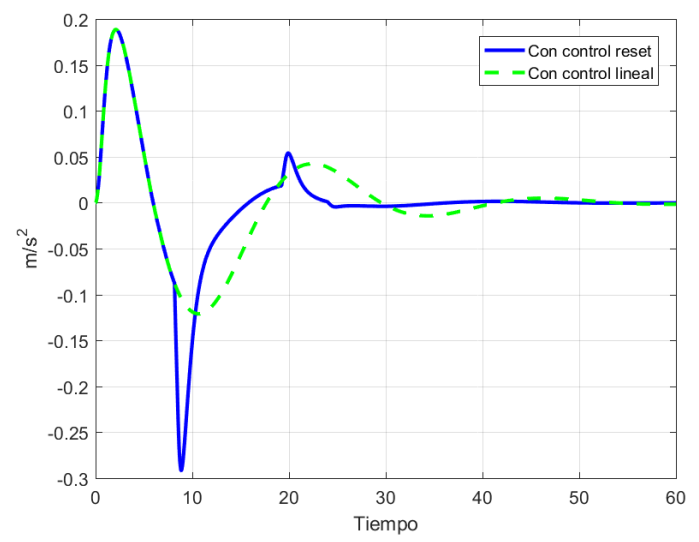

Figura 15: Componente Y (lateral) de la aceleración del centro de masas del vehículo.

respectivamente. Como se detalla en [23] y en el apartado 3.1 , los valores obtenidos para la maniobra de cambio de carril no superan los valores límite en ninguno de los dos casos.

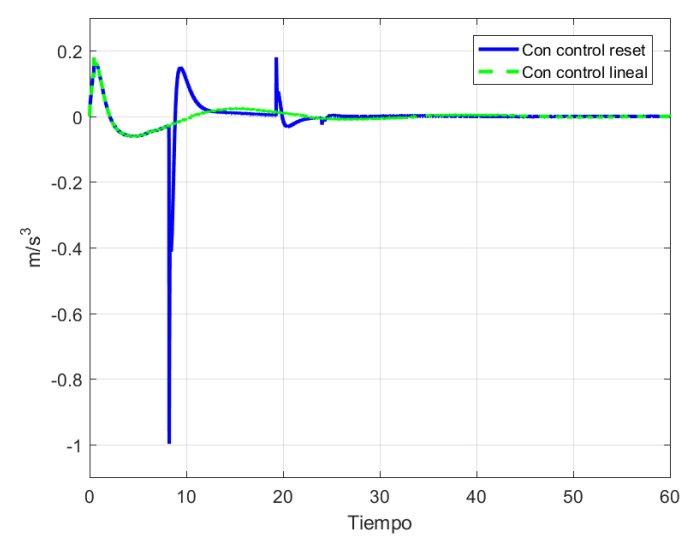

Figura 16: Componente Y (lateral) del jerk del centro de masas del vehículo.

\section{CONCLUSIONES Y LÍNEAS FUTURAS}

El objetivo de este trabajo ha sido explorar el potencial del control reset para una maniobra de cambio de carril comparando su rendimiento con el de un controlador lineal. Para hacer esto, se ha empleado un modelo sencillo para la sintonización del controlador. Se ha podido concluir que la aplicación del control reset permite obtener un regulador con mejor respuesta en régimen transitorio que el lineal y que igualmente presenta estabilidad robusta, siempre respetando los límites de confort para los ocupantes. CarSim ha sido utilizado tanto para la validación del modelo como para la del controlador y de su uso se desprende que el vehículo responde bien ante saltos en la señal de actuación (instantes de reinicio) y que por tanto, sería implementable en un vehículo real.

En cuanto a las líneas futuras se tratará de trabajar sobre el modelo dinámico del coche, ya que el modelo cinemático es válido a bajas velocidades y debe ser estimado para cada velocidad de funcionamiento. Otra modificación será añadir la actuación directamente sobre el volante de dirección del vehículo, lo que hará más realista la aplicación con la introducción de los correspondientes retardos de actuación del propio vehículo.

\section{Agradecimientos}

Este trabajo está financiado por MINECO, España, proyectos DPI2013-47100-C2-2-P y DPI2016-79278-C2-2-R y fondos FEDER.

\section{Referencias}

[1] Ni, L., Gupta, A., Falcone, P., Johannesson, L. (2016). Vehicle Lateral Motion Con- 
trol with Performance and Safety Guarantees. IFAC-PapersOnLine, 49(11), 285-290.

[2] Naranjo, J. E., Gonzalez, C., Garcia, R., De Pedro, T. (2008). Lane-change fuzzy control in autonomous vehicles for the overtaking maneuver. IEEE Transactions on Intelligent Transportation Systems, 9(3), 438-450.

[3] Hatipoglu, C., Ozguner, U., Redmill, K. A. (2003). Automated lane change controller design. IEEE transactions on intelligent transportation systems, 4(1), 13-22.

[4] Hahn, S., Zindler, K., Jumar, U. (2016). TwoDegrees-of-Freedom Lateral Vehicle Control using Nonlinear Model Based Disturbance Compensation. IFAC-PapersOnLine, 49(11), 182-189.

[5] Clegg, J. C. (1958). A nonlinear integrator for servomechanisms. Transactions of the American Institute of Electrical Engineers, Part II: Applications and Industry, 77(1), 41-42.

[6] Horowitz, I. (1982). Quantitative feedback theory. In IEE Proceedings D-Control Theory and Applications (Vol. 129, No. 6, pp. 215226). IET.

[7] Horowitz, I., Rosenbaum, P. (1975). Nonlinear design for cost of feedback reduction in systems with large parameter uncertainty. International Journal of Control, 21(6), 9771001 .

[8] Seron, M. M., Braslavsky, J. H., Goodwin, G. C. (2012). Fundamental limitations in filtering and control. Springer Science and Business Media.

[9] Åström, K. J. (2000). Limitations on control system performance. European Journal of Control, 6(1), 2-20.

[10] Baños, A., Vidal, A. (2007, June). Design of PI+ CI Reset Compensators for second order plants. In Industrial Electronics, 2007. ISIE 2007. IEEE International Symposium on (pp. 118-123). IEEE.

[11] Baños, A., Barreiro, A. (2011). Reset control systems. Springer Science and Business Media.

[12] Costas, A., Cerdeira-Corujo, M., Barreiro, A., Delgado, E., Baños, A. (2016, September). Car platooning reconfiguration applying reset control techniques. In Emerging Technologies and Factory Automation (ETFA), 2016 IEEE 21st International Conference on (pp. 1-8). IEEE.
[13] Costas, A., Cerdeira-Corujo, M., Barreiro, A., Delgado, E., Baños, A. (2016). Control basado en Reset para seguimiento de consigna en el sistema de Control de Crucero Adaptativo. Jornadas de Automática.

[14] Cerdeira-Corujo, M., Costas, A., Delgado, E., Barreiro, A., Baños, A. (2016). Gainscheduled wheel slip reset control in automotive brake systems. In Power Electronics, Electrical Drives, Automation and Motion (SPEEDAM), 2016 International Symposium on (pp. 1255-1260). IEEE.

[15] Cerdeira-Corujo, M., Costas, A., Delgado, E., Barreiro, A. (2017). Comparative Analysis of Gain-Scheduled Wheel Slip Reset Controllers with Different Reset Strategies in Automotive Brake Systems. In CONTROLO 2016 (pp. 751-761). Springer International Publishing.

[16] Beker, O. (2001). Analysis of reset control systems. Ph.D. thesis, Department of Electrical and Computer Engineering, University of Massachusetts, Amherst, MA.

[17] Aström, K. J., Murray, R. M. (2010). Feedback systems: an introduction for scientists and engineers. Princeton university press.

[18] Rajamani, R. (2011). Vehicle dynamics and control. Springer Science and Business Media.

[19] CarSim. Mechanical Simulation Corporation. Descripción general, http://carsim.com/ products/carsim/index.php, 2017.

[20] Kinjawadekar, T., Dixit, N., Heydinger, G. J., Guenther, D. A., Salaani, M. K. (2009). Vehicle dynamics modeling and validation of the 2003 Ford Expedition with ESC using CarSim (No. 2009-01-0452). SAE Technical Paper.

[21] Benekohal, R. F., Treiterer, J. (1988). CARSIM: Car-following model for simulation of traffic in normal and stop-and-go conditions. Transportation research record, (1194).

[22] Freudenberg, J. S., Middleton, R. H., Braslavsky, J. H. (1995). Inherent design limitations for linear sampled-data feedback systems. International Journal of Control, 61(6), $1387-1421$

[23] Chee, W., Tomizuka, M. (1994, June). Lane change maneuver of automobiles for the intelligent vehicle and highway system (IVHS). In American Control Conference, 1994 (Vol. 3, pp. 3586-3587). IEEE. 